\title{
APLICAÇÃO DO MODELO PSP MANUAL E AMPARADO POR FERRAMENTA CASE EM UM ESTUDO DE CASO DE FÁBRICA DE SOFTWARE BRASILEIRA
}

\section{APPLICATION Of MODEL PSP MANUAL AND SUPPORTED BY TOOL MARRIES IN A STUDY OF CASE OF BRAZILIAN PLANT OF SOFTWARE}

\author{
Denis Ávila Montini \\ Professor Mestre em Engenharia de Produção \\ Universidade Paulista - Engenharia de Produção \\ Rua Dr. Bacelar 1212 - Térreo - CEP 04026-002 - São Paulo - SP. \\ denisavilamontini@yahoo.com.br \\ Mauro de Mesquita Spinola \\ Professor Doutor Engenharia de Produção \\ Universidade Paulista - Engenharia de Produção \\ Rua Dr. Bacelar 1212 - Térreo - CEP 04026-002 - São Paulo - SP. \\ mauro@spinola.eng.br \\ José Benedito Sacomano \\ Professor Doutor Engenharia de Produção \\ Universidade Paulista - Engenharia de Produção \\ Rua Dr. Bacelar 1212 - Térreo - CEP 04026-002 - São Paulo - SP. \\ sacomano@terra.com.br

\section{Marcos Nascimento} \\ Professor Doutorando Engenharia de Produção \\ PSF - Pesquisadores sem Fronteira \\ Rua Augusto Perroni $6061^{\circ}$ And.- 05539-020 Butantã - São Paulo - SP. \\ marcos@psf.org.br

\section{Danilo Battaglia} \\ Analista Consultor - Pesquisadores sem Fronteira \\ PSF - Pesquisadores sem Fronteira \\ Rua Augusto Perroni $6061^{\circ}$ And.- 05539-020 Butantã - São Paulo - SP. \\ Danilo.battaglia@terra.com.br
}


Em um contexto de melhoria contínua de qualidade em projetos de desenvolvimento de software é aplicado experimentalmente o processo PSP para disciplinar alguns dos processos sugeridos pelo CMMI nível 2 com duas estratégias distintas. A primeira consiste em observar o comportamento de uma fábrica de software colhendo os dados necessários para atender o modelo PSP manualmente e na segunda visão a coleta ocorreu com o amparo de uma ferramenta CASE. Os resultados alcançados apontam os impactos nos rendimentos e nos padrões de qualidade que as duas estratégias proporcionaram e com os seus pontos fortes e as suas vulnerabilidades. Em ambos os casos o cumprimento dos prazos foi conseguido a partir do momento em que a especificação e o andamento das atividades foram controlados pelas práticas sugeridas pelo PSP.

Palavras chave: CMMI, PSP, CASE, Fábrica, Melhoria de processos.

\begin{abstract}
In a context of continuous improvement of quality in software development's projects, the PSP experimental process was applied to discipline some of the processes suggested by CMMI level 2 with two different strategies. The first one consists of observing the behavior of a software factory on collecting the necessary data to assist the PSP model manually, and in the second one the collecting happened with the help of a CASE tool. The results show the impacts in the performance and in the quality patterns that the two strategies provided with their advantages and their vulnerabilities. In both cases the fulfillment of the stated periods was obtained from the moment where the specification and the course of the activities were controlled by the two PSP' strategies suggested.
\end{abstract}

Key words: CMMI, PSP, CASE, Factory, Improvement of processes.

\title{
1. OBJETIVO
}

Este artigo se propôs a apresentar uma utilização do PSP[1] em um processo brasileiro de fábrica de desenvolvimento de software. O uso desta prática pode ser um bom ponto de partida para a viabilização do CMMI na gestão integrada de projeto. A pesquisa está centrada na customização da metodologia PSP, buscando o atendimento dos requisitos do nível 2 do CMMI de uma linha de produção de software. Um estudo de caso foi realizado em um lote de programas que foram encomendados por um determinado cliente.

O ponto relevante deste estudo é apresentar uma experiência na análise de desempenho de um processo de engenharia de software utilizando duas estratégias, uma com a tomada de dados manuais e outra amparada por ferramenta CASE visando obter êxito no gerenciamento básico de projeto. Em ambos os casos o cumprimento dos prazos foi conseguido a partir do momento em que a especificação e o andamento das atividades foram controlados pelas práticas sugeridas pelo PSP.

\section{A ORGANIZAÇÃO DO TRABALHO}

O trabalho possui oito seções dispostas para apresentar o conceito do PSP no acompanhamento no ciclo de desenvolvimento de software. Com esta diretriz, começamos com a seção $\mathbf{1}$ onde apresentamos o objetivo do trabalho e sua organização na seção 2; na seção $\mathbf{3}$ contextualizamos o 
ambiente de desenvolvimento de uma fábrica de software brasileira. Na seqüência, na seção 4 delimitamos a atuação do estudo e na seção 5, inserimos uma proposta de implementação do PSP enfocando o controle manual do processo PSP, para se obter ganho de escala com a metodologia. $\mathrm{Na}$ seção 6 começa a descrição do comportamento do uso de uma ferramenta CASE.

Na seção 7 são comparados criticamente os resultados obtidos no arranjo proposto, descrevemos as análises e os resultados. Na seção 8 é apresentada a conclusão do caso estudado e são sugeridas algumas variações a serem exploradas em estudos complementares.

\section{INTRODUÇÃO}

A indústria de software no Brasil tem uma história recente, e as suas atuais características estruturais refletem a sua dinâmica competitiva. A partir dos diversos estudos realizados sobre esta indústria, pode-se dividir a sua evolução em basicamente dois períodos: $1^{\circ}$ antes de 1990 , em um contexto de substituição de importações; e $2^{\circ}$ a partir de 1990, em um contexto de competição global [SOFTEX, 02].

Desde o começo do ano de 2004 a empresa AML[2] estava em processo de implantação do programa CMMI[3] para melhorar suas competências e seus requisitos formais que um cliente da área financeira estabeleceu para os seus fornecedores. Um grupo de estudo de CMMI no início das atividades decidiu adotar as práticas do PSP [PSF, 06] para disciplinar os processos de desenvolvimento. $\mathrm{O}$ grupo coletou dados referentes às técnicas e práticas aplicadas com a finalidade de contemplar os requisitos do nível 2 do modelo CMMI.

A abordagem incremental do desenvolvimento de software foi formulada por Watts Humphrey como um conjunto de regras [PEDRYCZ, 03] que permitem que o monitoramento do processo de desenvolvimento seja amparado por métricas. A pesquisa seguiu os indicadores especificados de Hunphrey em seu modelo evolutivo.

Segundo Sommerville [SOMMERVILLE, 03] o PSP[4] constitui-se como sendo um processo de trabalho pessoal que colabora no planejamento das atividades do desenvolvimento sendo que para isto utiliza-se um sistema de informações amparado por formulários. Antes de chegarmos a este ponto, o núcleo de projeto teve de tomar algumas decisões sobre a sua atuação nos processos. Porém quais seriam as variáveis envolvidas neste tipo de definição?

\section{A IMPORTÂNCIA DA DISCIPLINA EM PROCESSOS DE PLANEJAMENTO}

A engenharia de software apresenta métodos e ferramentas para permitir a produção de software de maneira econômica, dentro dos prazos visando aumentar a escala [PRESSMAN, 02]. A satisfação dos requisitos acordados com o cliente tem que estar claramente assimilado pela equipe do projeto, onde a assertividade do prazo é uma das metas e é um dos fundamentos da engenharia de software.

Neste contexto, têm surgido ao longo dos anos, inúmeras propostas de metodologias e ferramentas visando auxiliar o processo de desenvolvimento do software. A equipe de projeto deve determinar um modelo de trabalho que interaja com as várias funções da organização integrando as equipes, etapas e processos para viabilizá-la na sua produção.

Para a realização deste estudo isolamos as etapas de codificação e testes onde escolhemos um modelo piloto para estabelecer um controle em cada etapa do desenvolvimento visando coletar dados de cada atividade realizada. Com a estabilização deste sistema manual inicial alguns aspectos definidos do nível dois, repetido do modelo CMMI, começaram a estabilização do planejamento e acompanhamento de projetos. 
O acompanhamento da evolução do projeto através da tomada de dados é fundamental para a manutenção dos prazos e correções do planejamento. A equipe de engenharia de software, ao atender as diretrizes do CMMI, dependeria do uso efetivo de práticas individuais assim como pessoas e ferramentas. O PSP contribui com a disciplina para fundamentar as atividades que a engenharia precisa para estabelecer os meios de como produzir artefatos de qualidade usando princípios individuais de aperfeiçoamento de processo.

\section{O PSP EM UMA FÁBRICA DE SOFTWARE CMMI NÍVEL 2}

Para atingir o ambiente de desenvolvimento confiável e disciplinado, a equipe de engenharia precisou de uma estratégia de apoio que se constituiu na conscientização da equipe e no preenchimento formal dos formulários que a prática requer.

O CMMI e o PSP se complementam e para viabilizar o aperfeiçoamento do processo organizacional integrando as duas visões, é necessário realizar exercícios individuais com o apoio da engenharia da fábrica em elementos comuns à duas visões. No quadro 1 apresentamos uma tabela com alguns destes elementos.

\begin{tabular}{||l|l|l|l||}
\hline \multicolumn{3}{|c|}{ Técnicas de Melhoria do Processo de Software } \\
\hline \multicolumn{1}{|c|}{ Métodos Características } & \multicolumn{1}{|c|}{ PSP } & \multicolumn{1}{|c|}{ CMMI@ } & \multicolumn{1}{|c|}{ ISSO 9000:2001 } \\
\hline Propósito & $\begin{array}{l}\text { Gerenciamento e Melhoria } \\
\text { da Qualidade }\end{array}$ & $\begin{array}{l}\text { Gerenciamento e Melhoria } \\
\text { da Qualidade }\end{array}$ & $\begin{array}{l}\text { Gerenciamento da } \\
\text { Qualidade }\end{array}$ \\
\hline Tempo & Muito curto & Breve & Tarde \\
\hline Recomendação & Explicito & Descritivo & Descritivo \\
\hline Definição & Exato & Vago & Vago \\
\hline Usuários & Gerentes e Engenheiros & Gerentes e Engenheiros & Gerentes \\
\hline Cobertura & Ciclo de vida do software & $\begin{array}{l}\text { Gerenciamento do Projeto } \\
\text { (estágios, contínuos) }\end{array}$ & Garantia da Qualidade \\
\hline Custo & Muito baixo & Alto & Alto \\
\hline Qualidade & Muito Alto & Médio & Baixo \\
\hline Duração & Semanas & Anos & Anos \\
\hline Ponto de Equilibro & Horas & Anos & Anos \\
\hline Escopo & Holístico & Ambíguo & Baixo \\
\hline Mensuração & Muito alto & Médio \\
\hline \hline
\end{tabular}

Quadro 1 - Técnicas de Melhoria do Processo de Software - Adaptado de [HUMPHREY, 00] e [STROMBERG, MUTAFELIJA, 03].

Esta escolha auxiliada por um gerenciamento de ações específicas onde às pessoas envolvidas transmitiram o conhecimento adquirido com o projeto para a empresa, no primeiro projeto teve o desempenho prejudicado nos primeiros dias até a assimilação e entendimento dos participantes com as conscientizações do preenchimento correto dos formulários. Já a partir do segundo projeto o desempenho foi estabilizado com o processo entendido e assimilado por todos.

Para que se estabilize o desempenho esperado, primeiramente foi realizada a montagem de uma base de dados para receber os dados dos formulários preenchidos pelos analistas.Finalizada a montagem, começou o abastecimento da base de dados de projeto, viabilizando o dimensionamento da produção da equipe e possibilitando as análises realizadas pela engenharia.

Com este aprendizado, a calibragem da capacidade da equipe e o subseqüente conhecimento gerado sobre o seu comportamento, embasado em dados históricos, proporcionaram planejamentos mais realistas. A relevância da atividade está na melhoria das estimativas que os novos projetos receberam e a diminuição do risco ocorreu devido ao caráter científico que os planejamentos passaram a ter. Para 
aportar a estratégia da diminuição do risco, do acompanhamento e do planejamento de projetos, optouse pela escolha do processo do PSP.

A composição deste modelo manual de processo é aderente aos requisitos da melhoria contínua da ISO [ABNT, 03] e PSP [HUMPHREY, 94] em seus procedimentos. Os usos adequados dos processos facilitam a padronização e a sua normalização. A ISO [ABNT, 03] é aderente aos fundamentos da visão evolucionista do CMMI, pois os dois modelos trabalham a visão da melhoria continua das atividades.

Para a implantação inicial do PSP, sabendo que este é dividido em sete níveis conforme detalhado no quadro 2, Comparativo dos níveis do PSP com CMMI, foi escolhida uma forma de implantação incremental. Nos projetos as equipes conseguiram na íntegra o nível do PSP 0 e parcialmente o PSP 0.1. Como os níveis superiores adicionam características aos níveis já implantados a evolução do processo é cumulativa e não há perda de tempo ou redundância de atividades. A equipe apenas precisa se adaptar as novas técnicas solicitadas.

\begin{tabular}{|l|l|l|l||}
\hline \multicolumn{1}{|c|}{ Nome do Processo } & CMMI nível 2 & \multicolumn{1}{|c||}{ Nível } & \multicolumn{1}{c||}{ Atividade } \\
\hline Processo de Medição Pessoal & PMC M\&A & PSP 0 & $\begin{array}{l}\text { Registro de Tempo, Registro de Defeitos e } \\
\text { Padrão de tipos de defeitos }\end{array}$ \\
\cline { 2 - 4 } & PMC M\&A & PSP 0.1 & $\begin{array}{l}\text { Padrão de Codificação, Medida de Tamanho, } \\
\text { Proposta de Melhoramento de Processo. }\end{array}$ \\
\hline Processo de Planejamento Pessoal & PMC M\&A & PSP 1 & Estimativa de Trabalho e Relatório de Testes \\
\hline PMC PPQA & PSP 1.1 & Planejamento de Tarefas e Cronogramas \\
\hline $\begin{array}{l}\text { Processo de Gerenciamento de } \\
\text { Qualidade Pessoal }\end{array}$ & PMC PPQA & PSP 2 & Revisões de Código e Revisões de Projeto \\
\hline Processo Pessoal Cíclico & PMC PP & PSP 2.1 & Padrões de Projeto \\
\hline \hline
\end{tabular}

Quadro 2 - Comparativo dos níveis do PSP com CMMI - Adaptado de [HUMPHREY, 00][5].

Os colaboradores envolvidos no desenvolvimento utilizam o PSP (insumos-scripts de orientação; etapas - planejamento; design; codificação; compilação, testes; postmortem; produtos gerados - $\log ^{\prime} s$ de traceabilidade e planejamento sumário das atividades), para disciplinar as atividades globais planejadas buscando implementar um programa de melhoria do processo de software sem a preocupação da certificação. O processo manual é trabalhoso, porém fundamental para a constituição da maturidade da empresa. Cada colaborador precisa ser treinado e monitorado pelo engenheiro de software (Software Engineering Process Group - SEPG). A limitação do processo manual está na capacidade humana de coletar, tabular e analisar os dados, logo o próximo passo para viabilidade desta informação para que a gerencia evite a perda de recursos e acelerar o processo com a automação.

\section{O GANHO EM ESCALA COM O USO DA FERRAMENTA CASE}

O objetivo de integrar o processo de documentos e artefatos deste sistema de informação é fundamental para oferecer um serviço ágil e com um desempenho melhor em relação ao processo convencional. A integração dos resultados da equipe propicia as análises de risco que o PSP precisa para refletir às diretrizes das áreas PPQA, PP, PMC e M\&A do CMMI nível 2 [RASMUSSEN, 03]. O sistema é justificável e proporciona a facilidade na realização das análises, acesso aos documentos e a coleta dos dados nos quais os formulários que o PSP necessita.

A ferramenta escolhida para o estudo foi a Innovative Suite 1.0 [INNOVATIVE, 04] que comporta todas as métricas sugeridas pelo PSP e organiza os processos de desenvolvimento de software. Para a constatação significativa do controle foi realizada a primeira etapa que consistiu no treinamento dos 
analistas e do pessoal envolvido no processo de desenvolvimento visando atender as solicitações dos gestores sobre o andamento das atividades. O sistema de controle funcionou através do abastecimento dos dados durante a operação pelos colaboradores treinados. As informações que antes eram preenchidas no questionário nesta etapa foram abastecidas diretamente na ferramenta. O SEPG passou a ter uma posição atualizada ao final de cada turno de trabalho devido ao fato de que a ferramenta tem um módulo de tratamento para a interpretação em tempo real das respostas.

\section{OS RESULTADO OBTIDOS COM O USO DOS PROCESSOS}

A diferença entre a aplicação manual do processo PSP e a utilização da CASE para apoiar o processo de desenvolvimento está na avaliação das capacidades de produção da equipe de desenvolvimento de software. Os diferenciais foram os rendimentos nas possíveis comparações e similaridades dos resultados entre os métodos. A equipe que trabalhou manualmente com os questionários, a adequação ao uso foi mais fácil em relação à adequação que as equipes que utilizaram a automação. $\mathrm{O}$ uso da ferramenta CASE não é intuitivo e precisa de um treinamento mais sofisticado para disciplinar o uso do método. Em ambos os casos os colaboradores precisam de treinamentos para operar os métodos e identificamos que em ambos os métodos aplicados identificaram-se nos processos pontos fortes e oportunidades de melhoria.

O processo manual é mais fácil de assimilar e é mais trabalhoso, no caso da ferramenta é justificado o esforço quando a empresa tende a realizar as operações diversas vezes, e precisa de resultados apurados pela criticidade do processo, pois a informação é mais ágil, porém é mais custoso e demorado para se implementar o treinamento e só se justifica a partir de um certo tamanho de software. A preocupação inicial com os resultados foi o impacto obtido com a apuração e o processamento dos dados obtidos. Então apresentaremos a tabulação dos dados utilizados nos processos manuais e eletrônicos. O sucesso parcial da técnica que visava o controle e melhoria na estimativa em ambos os casos foi obtida pela empresa mesmo com diversos fatores adversos. No quadro 3 apresentamos o resultado alcançado com o uso dos modelos:

\begin{tabular}{|c|c|c|c|c|}
\hline Equipe & Desenvolvimento & \multicolumn{2}{|c|}{ Qualidade } \\
\hline Atividade & $\begin{array}{c}\text { Programação: } \\
\text { Total de horas utilizadas }\end{array}$ & $\begin{array}{c}\text { Testes: } \\
\text { Total de horas } \\
\text { utilizadas }\end{array}$ & $\begin{array}{c}\text { Números de pontos de } \\
\text { controle }\end{array}$ & $\begin{array}{c}\text { Números de não } \\
\text { conformidades }\end{array}$ \\
\hline Previsão & 297 & 72 & 236 & 102 \\
\hline Consumo & 313 & 53,5 & 236 \\
\hline
\end{tabular}

Quadro 3 - Resultados obtidos.

Com a base de dados definida foi possível verificar outro tipo de informação sobre o comportamento da equipe de desenvolvimento no que tange ao seu desempenho. Dos resultados obtidos podemos deduzir este conjunto de análises a seguir:

\section{$\checkmark \quad 22,9 \%$ das horas de desenvolvimento foram dedicadas aos testes. \\ $\checkmark \quad 43,2 \%$ dos pontos de controles do desenvolvimento tiveram não-conformidades. \\ $\checkmark 1$ não-conformidade a cada 2,3 pontos de controles.}

A identificação das distorções entre o estimado e o realizado foi corrigida durante a operação, injetando recursos para assegurar a data de entrega. A diferença de habilidade entre os recursos que acabam gerando uma distorção significativa no planejamento. Os dados foram analisados e interpretados gerando um resultado que pode antecipar problemas e utilizar os recursos para minimizar alguns dos efeitos do não cumprimento dos prazos de entrega ou multa por atraso. Após esta avaliação a empresa passou a ter conhecimento do comportamento das suas variáveis de projeto, antes com o 
pré-projeto, durante com o acompanhamento e depois com as análises e o cadastro dos componentes para a sua reutilização. O processo manual foi mais fácil de se utilizar para projetos de até 100 pontos de função. A ferramenta CASE passa a ganhar escala e se justificar a partir desde ponto, pois possibilita o cruzamento de informações e previsões que o processo manual demoraria semanas para realizar.

O diferencial encontrado pela equipe de engenharia reside no fato de que os dois métodos que apoiam o processo PSP forneceram saídas de qualidade e de custo e prazo diferentes com resultados semelhantes. Cada um com sua vantagem específica possibilitando um leque de opções para os engenheiros na hora de realizar a escolha de qual método é o mais adequado para a situação em análise. Logo o ganho está nas variações estratégicas para a implementação de projeto onde a empresa passa a contar com mais este grau de adaptabilidade relevante com se está participando de uma licitação.

\section{CONCLUSÃO}

O método do PSP pode proporcionar para a gestão um aumento de informações se os colaboradores receberem treinamento e monitoração adequados. O êxito pode ser alcançado com a disciplina do método do PSP se os usuários estiverem treinados no processo e seguidos do acompanhamento do abastecimento dos repositórios. $\mathrm{O}$ andamento do processo passou a estar à disposição do gestor como um recurso confiável para a realização de estimativas com o conhecimento das informações subjetivas que as métricas tornaram visíveis. Os usos destas técnicas para reutilizar informações em novos projetos são facilitados quando novos projetos estimados tiverem características em comuns com as já armazenadas na base histórica. Quando as pessoas envolvidas tiveram o conhecimento das métricas e da sua importância desenvolvida, onde a preparação individual é fundamental, as estimativas passaram a estar mais próximas do real com o reuso deste tipo de informações. Os cuidados tomados para a análise comparativa foram escolhidos de lotes de programas que tinham as mesmas características de extensão, prazo para se obter um agrupamento comum. A pesquisa obteve o êxito de colher os dados de seus projetos de acordo com os formulários do PSP [HUMPHREY, 00].

A empresa teve que repensar sua forma de trabalho para melhorar as estimativas para os próximos lotes. O ponto-chave do estudo consiste então no entendimento do impacto que uma ferramenta pode dar quando esta se propõe a agilizar o conhecimento dos dados e informações durante o processo. Analisamos que a tomada de decisão pode constituir um elemento de planejamento para medir a capacidade produtiva da equipe, ações interativas, pró-ativas e de correções para viabilizar a contingência assegurando a retomada da confiabilidade.

O principal benefício da sistematização foi facilitar o processo de avaliação que a empresa passa ao realizar um determinado turno de trabalho, sendo este controle fundamental para a certificação do CMMI. Para isto é preciso que todas as pessoas envolvidas tenham um treinamento prévio para a realização das atividades. A gestão do projeto só poderá ser bem sucedida se após o levantamento de requisitos for suficientemente claro para que a empresa possa comparar as distorções entre o contratado e entregue. A empresa precisa especializar o seu controle sobre o método, pois o volume de dados e informações pode ser extenso para que somente um analista, sem o apoio de um instrumento e sem uma infra-estrutura, possa consolidar os dados para os gestores. O processo de análise leva tempo e precisa ser planejado para que a ação corretiva em tempo hábil possa ser viabilizada.

O PSP tanto manual quanto amparado por ferramentas fornece uma estrutura conceitual para a melhoria da gestão e do desenvolvimento de produtos de software de uma forma consistente e disciplinada. Ele não garante que os produtos de software serão construídos com sucesso ou que todos 
os problemas de desenvolvimento de software serão isolados e resolvidos adequadamente. A composição das visões do PSP com a do CMMI são complementares.

Ao realizar este estudo de caso com a metodologia PSP para processos de desenvolvimento de software, percebe-se que a proposta de viabilizar a identificação da vulnerabilidade em processo de controle da produção de software é possível.Depende, porém, do nível de consciência da equipe e da capacidade de treinamento que a empresa aplica na transmissão dos conhecimentos necessários para que se possa operacionalizar a sua maturidade de operação. As aquisições de dados honestos formarão à base da organização sistêmica. A manipulação dos erros que acarretam problemas não tratados neste estudo. O uso do método acompanhado das calibrações ameniza as distorções e os riscos dos problemas tendem a estar sobre controle sendo abordados de forma mais bem estruturada.

\section{NOTAS DE FINAL DE TEXTO}

[1] PSP - Personal Software Process - Processo de Software Pessoal - que se constituí como sendo um processo disciplinado de auto melhoria para desenvolvimento de software. Watts $S$. Humphrey, The Personal Process in Software Engineering, Software Engineering Institute, Carnegie Mellon 1994.

[2] AML System Ltda. Empresa de engenharia e desenvolvimento de Software. Rua Vergueiro, 1855 - $4^{\circ}$ Andar - Paraíso - São Paulo - SP - 04101-000. Fone: (0xx1 1) 5572-7007 - R.236

[3] O Instituto de Engenharia de Software, Software Engineering Institute, SEI, desenvolveu a sua visão sobre o modelo de maturação de projeto de desenvolvimento de software, o modelo de capacitação e maturidade, CMM, Capability and Maturity Model, e o modelo de capacitação e maturidade integrado, CMMI-CMM Integrated. A diferença entre os dois é a integração com a gerência de projeto fortemente explorada na CMMI.

[4] O PSP é um framework de formulários, diretrizes e procedimentos de desenvolvimento de software. O PSP corretamente usado provê os dados históricos para que o engenheiro de software possa conhecer melhor seus compromissos, tornar os elementos rotineiros de seu trabalho mais previsíveis, confiáveis e possibilitar a medição da qualidade dos produtos gerados. Adaptado de [HUMPHREY, 00].

[5] Notas dos autores: As áreas de processos do CMMI nível 2 envolvidos neste estudo: PMC Product Monitoring and control - Controle e monitoração de produto, M \&A -Measurement and Analysis - Análise e medição, PPQA - Process and Product Quality Assurance - Garantia da Qualidade de produtos e processos, PP - Project Planning - Planejamento e projeto [RASMUSSEN, 03] Rasmussen, Karen, STBC Mapping CMMI-SE/SW Software Engineering Institute (SEI) - Instituto de Engenharia de Software - Encontrado no site: http:// www.sei.cmu.edu/cmmi/adoption/stsc-mappings.pdf

[6] A Innovative Suíte 1.0 é aplicada a engenharia de software com um compromisso de qualidade no projeto e integração com todo o ciclo de desenvolvimento do produto. Av. Barbosa Lima 149 Sala 217 - Bairro do Recife, Recife-PE Brasil - CEP 50330-030. - Tel. (55-81) 3424.6206 Encontrado no site: innovative@innovative.inf.br. 


\section{REFERÊNCIAS}

[ABNT, 03] NBR ISO 9000:200, Sistemas de Gestão da qualidade - Diretrizes para a melhoria de desempenho. Encontrada na Associação Brasileira de Normas Técnicas São Paulo em http://www. abnt. org. br. ABNT, 2003.

[HUMPHREY, 94] Watts S. Humphrey, The Personal Process in Software Engineering, Software Engineering Institute (SEI), Carnegie Mellon 1994.

[HUMPHREY, 00] Humphrey, Watts S, TECHNICAL REPORT CMU/SEI-2000-TR-022, Software Engineering Institute - Encontrado no site: http: //www.sei.cmu.edu/publications/documents /00.reports/00tr022.html - SEI-2000.

[INNOVATIVE, 04] Innovative Suíte 1.0, Suite de Engenharia de software, Encontrado no site: innovative@innovative.inf.br - Innovative, 2004.

[PEDRYCZ, 03] James F. Peters/ Witold Pedrycz Engenharia de Software Rio de Janeiro, Editora Campus, 6a edição. 2001.

[PRESSMAN, 02] PRESSMAN, S. Roger, Engenharia de Software São Paulo, Editora Mc Graw Hill Wesley, $5^{\circ}$ edição, 2002.

[RASMUSSEN,03] Rasmussen, Karen, STBC Mapping CMMI-SE/SW Software - Engineering Institute (SEI) - Instituto de Engenharia de Software - Encontrado no site: http:// www.sei.cmu.edu/cmmi/adoption/stsc-mappings.pdf - SEI, 2003.

[SOMMERVILLE, 03] SOMMERVILLE, Ian, Engenharia de Software São Paulo, Editora Addison Wesley, 6a edição, 2003.

[STROMBERG,MUTAFELIJA,03] Stromberg, Harvey, Mutafelija, Boris, ISO-9901:2000 - CMMI V 1.1 Mappings CMMI-SE/SW Software Engineering Institute (SEI) - Instituto de Engenharia de Software - Encontrado no site: http://www.sei. cmu.edu/cmmi/adoption/iso-mapping.pdf SEI, 2003.

[SOFTEX, 02] A indústria de software no Brasil 2002: fortalecendo a economia do conhecimento - Massachussets Institute of Technology - MIT; Brasil Coordenação geral Sociedade SOFTEX. - Campinas: SOFTEX, 2002.

[PSF, 06] $\quad$ www.psf.org.br, Pesquisadores sem fronteira - ONG de suporte a pesquisa científica do estado de São Paulo, Brasil. 2006.

Artigo recebido em 18/07/05 e aceito para publicação em 07/08/06 